\title{
Prevalence, Etiology, and Outcome of Sepsis among Critically III Patients in Malawi
}

\author{
Meghan Prin, ${ }^{1 *}$ Lauren Onofrey, ${ }^{2}$ Laura Purcell, ${ }^{3}$ Clement Kadyaudzu, ${ }^{4}$ and Anthony Charles ${ }^{3}$ \\ ${ }^{1}$ Department of Anesthesiology, University of Colorado School of Medicine, Aurora, Colorado; ${ }^{2}$ Department of Internal Medicine, University of \\ Washington School of Medicine, Seattle, Washington; ${ }^{3}$ Department of Surgery, University of North Carolina at Chapel Hill, Chapel Hill, North \\ Carolina; ${ }^{4}$ Department of Anesthesiology, Kamuzu Central Hospital, Lilongwe, Malawi
}

\begin{abstract}
There are scarce data describing the etiology and clinical sequelae of sepsis in low- and middle-income countries (LMICs). This study describes the prevalence and etiology of sepsis among critically ill patients at a referral hospital in Malawi. We conducted an observational prospective cohort study of adults admitted to the intensive care unit or high-dependency unit (HDU) from January 29, 2018 to March 15, 2018. We stratified the cohort based on the prevalence of sepsis as defined in the following three ways: quick sequential organ failure assessment (qSOFA) score $\geq 2$, clinical suspicion of systemic infection, and qSOFA score $\geq 2$ plus suspected systemic infection. We measured clinical characteristics and blood and urine cultures for all patients; antimicrobial sensitivities were assessed for positive cultures. During the study period, 103 patients were admitted and 76 patients were analyzed. The cohort comprised $39 \%$ male, and the median age was 30 (interquartile range: 23-40) years. Eighteen (24\%), 50 (66\%), and 12 patients (16\%) had sepsis based on the three definitions, respectively. Four blood cultures $(5 \%)$ were positive, two from patients with sepsis by all three definitions and two from patients with clinically suspected infection only. All blood bacterial isolates were multidrug resistant. Of five patients with urinary tract infection, three had sepsis secondary to multidrug-resistant bacteria. Hospital mortality for patients with sepsis based on the three definitions ranged from $42 \%$ to $75 \%$ versus $12 \%$ to $26 \%$ for nonseptic patients. In summary, mortality associated with sepsis at this Malawi hospital is high. Bacteremia was infrequently detected, but isolated pathogens were multidrug resistant.
\end{abstract}

\section{INTRODUCTION}

Most of the global burden of sepsis occurs in low- and middle-income countries (LMICs), ${ }^{1}$ but the prevalence and etiology of sepsis in LMICs are not well understood. In particular, the lack of laboratory infrastructure in many LMICs has historically precluded an assessment of the pathogens leading to sepsis. A recent systematic review found that data describing antimicrobial resistance were absent for $43 \%$ of countries in Africa, ${ }^{2}$ and only two countries have national antimicrobial resistance plans. ${ }^{3}$ In addition, small studies have identified indiscriminate antibiotic use both in and out of hospital settings in sub-Saharan Africa. ${ }^{4,5}$ The absence of microbiological data and lack of antibiotic stewardship complicate sepsis management and almost certainly worsens outcomes, particularly in low-resource systems. The purpose of this study was to examine the prevalence, etiology, and outcomes of sepsis among a cohort of critically ill patients in a referral hospital of Malawi, with a focus on the prevalence of culture-confirmed bacteremia and urinary tract infections.

\section{METHODS}

We conducted a prospective, observational cohort study of adults admitted to the intensive care unit (ICU) or adult highdependency units (HDUs) of Kamuzu Central Hospital $(\mathrm{KCH})$ in Lilongwe, Malawi, from January 29, 2018 to March 15, 2018. The sample size and study time period were determined by staffing and funding limitations. The study protocol was approved by the National Health Sciences Research Council of Malawi and the Institutional Review Boards of Columbia University College of Physicians and Surgeons, and the University of North Carolina at Chapel Hill, with which the

\footnotetext{
*Address correspondence to Meghan Prin, Department of Anesthesiology, University of Colorado School of Medicine, 12401 East, 17th Ave., 7th Floor, Aurora, CO 80045. E-mail: meghan.prin@
} cuanschutz.edu study was affiliated, and the requirement for written informed consent was waived by all ethics oversight bodies.

Study setting. Malawi is a country in southeast Africa with a population of 18 million people, a life expectancy of 63.8 years, and a Human Development Index rank of 170 of 187 countries. ${ }^{6}$ It is the sixth poorest country in sub-Saharan Africa. ${ }^{7}$ Kamuzu Central Hospital is a referral hospital in the central region of Malawi, with a catchment area of approximately five million. The ICU at $\mathrm{KCH}$ is a five-bed unit which offers a 1:1 nurse-to-patient ratio, continuous noninvasive vital sign monitoring, mechanical ventilation with a titratable fraction of inhaled oxygen, and intravenous medication infusions. Hemodialysis is available within the hospital if resources allow and if patients can be transferred to the nearby unit where it is provided. Clinical care in the ICU is directed by clinical officers in anesthesiology. No staff members have consultant-level expertise in intensive care medicine. There are three adult HDUs at $\mathrm{KCH}$, which are staffed by the services managing patients in each unit: medicine, surgery, and obstetrics and gynecology. All the HDUs have four beds each, a nurse-topatient ratio ranging from 1:2 to $1: 4$, continuous noninvasive vital sign monitoring, supplemental oxygen, and noninvasive respiratory support (continuous or bi-level positive airway pressure). The key difference between the ICU and the HDUs is the availability of mechanical ventilation. Clinical care in the ICU and HDU is provided free of charge to all patients, including all available medications as ordered by the medical staff. Laboratory measurements are generally not ordered unless paid for directly by patients.

Data collection. The study data were prospectively collected by research staff trained in ICU and HDU data abstraction. All patients admitted to the study ICU or HDUs during the recruitment period were eligible for inclusion. The exclusion criteria included patients aged $\leq 16$ years or the inability to collect clinical data (e.g., file missing and patient eloped). When patients were readmitted to the HDU or ICU or vice versa, the readmission was analyzed as part of their 
continuous hospital course rather than as an index admission for a new participant. The primary outcome was in-hospital mortality.

Data collection began for each patient at the time of the index ICU or HDU admission, at which time staff would review the medical files, order laboratory tests, and then follow up the patient through the hospital course. Variables collected included the date of hospital admission, hospital location before ICU or HDU (e.g., emergency room, operating room, and ward), vital signs and laboratory measurements at the time of admission to ICU, hospital discharge date, and vital status at hospital discharge. Laboratory investigations at the time of ICU or HDU admission for all patients in this study included a complete blood count, electrolytes, peripheral aerobic blood culture, mycobacterial culture, ${ }^{8,9}$ urine culture, point-of-care malaria status, and point-of care HIV status. These measurements are not the standard of care for patients outside the research context. Cerebrospinal fluid, pulmonary cultures, and anaerobic cultures were not taken secondary to funding and staff limitations.

There are protocols at $\mathrm{KCH}$ to ensure early and appropriate treatment of infections and sepsis, and providers in this setting maintain a high clinical suspicion for sepsis. For example, blood cultures are to be drawn on all patients who receive antibiotics while hospitalized. The implementation of this protocol varies depending on available resources. Our previous work has demonstrated that $60 \%$ of critically ill patients admitted to the ICU at $\mathrm{KCH}$ are suspected of having an infection. ${ }^{10}$ The clinical suspicion of systemic infection is based on staff interpretation of the history, vital signs, and physical exam. Although most patients suspected of infection receive intravenous antibiotics, laboratory resources are so limited that cultures are not usually drawn. Given the severely restricted diagnostic resources and the lack of validated tools for screening sepsis in this setting, we elected to draw blood and urine cultures on all patients rather than just those meeting the traditional sepsis criteria (e.g., definitions derived from high-income settings). For patients readmitted between the HDU and ICU, cultures were repeated if a new infection was suspected.

Laboratory methods. Urine cultures were drawn sterilely from indwelling urinary catheters (standard at admission in all HDU and ICU patients in this study site) and observed for 48 hours. A positive urine culture was defined by an organism other than common skin flora (e.g., Staphylococcus epidermidis) identified with a > 100,000 organisms/mL colony count. Urinary tract infection was defined as a positive urine culture in addition to fever during the ICU or HDU course. Fever was defined as a skin temperature of $>38.4^{\circ} \mathrm{C}$; we chose a relatively high cutoff to maintain specificity for fever in this hot tropical study site, where hospital wards are not temperature controlled. Blood cultures were drawn with an aseptic technique by a specially trained study staff member. Aerobic blood cultures were observed for 5 days, and mycobacterial blood cultures were observed for 5 weeks. Most organisms isolated in the blood were considered positive blood cultures; cultures positive for common skin flora and contaminants (coagulase-negative Staphylococcus) were examined with the laboratory leadership on a case-by-case basis, considering the clinical picture and the leukocyte count. For all cultures, the laboratory uses the Kirby and Bauer method to measure the in vitro susceptibility of bacteria to antimicrobial agents by agar disk diffusion. All aspects of the procedure are standardized as recommended by the Clinical and Laboratory Standards Institute in the document M100-S. ${ }^{11}$

Multidrug resistance was defined as non-susceptibility to at least one agent in three or more antimicrobial categories. ${ }^{12}$ The most commonly administered antibiotics for hospitalized patients in Malawi are ceftriaxone and metronidazole, based on the Malawi Standard Treatment Guidelines. ${ }^{13}$ Although this resource-limited study hospital does not have every antimicrobial that may be available in high-income settings, other antibiotics commonly available at $\mathrm{KCH}$ include ampicillin, chloramphenicol, ciprofloxacin, gentamicin, imipenem, and trimethoprim-sulfamethoxazole.

Data analysis. We first stratified the cohort based on the prevalence of sepsis, using several definitions based in the literature and applicable in this setting: quick sequential organ failure assessment (qSOFA) score $\geq 2$, clinically suspected systemic infection, and qSOFA $\geq 2$ plus clinically suspected systemic infection. ${ }^{14}$ We then described the cohort using these stratifications, including the demographics, the vital signs, the relevant laboratory values at ICU or HDU admission (e.g., leukocyte count and blood cultures), and in-hospital mortality. We assessed for differences in clinical characteristics between those with and without sepsis using the three definitions. We then performed multivariate analysis to assess risk factors for the primary outcome of hospital mortality. Finally, we reviewed the culture results and reported antimicrobial sensitivities for isolated organisms.

We summarized continuous variables using the median and interquartile range (IQR) and categorical variables by the proportion of each respective category. We used chi-square and Fisher's exact tests for categorical variables and the Student's $t$-test or analysis of variance for continuous variables, as appropriate. In multivariate analysis, we entered clinical variables into a logistic regression model and conducted backward selection based on a $P$-value of 0.2 . Leukocyte count was excluded from this model as it may not easily be measured in many low-income setting ICUs, as was respiratory rate which may have been confounded by the use of the ventilator. Statistical significance was defined as $P<$ 0.05 for univariate analyses and the final models. All statistical analyses were conducted using StataSE/14.2 (StataCorp, College Station, TX).

\section{RESULTS}

During the study period, 103 patients were admitted to the study ICU and HDUs, and, after exclusions, there were 76 patients for analyses. (Figure 1). Patients were primarily excluded for missing data (Supplemental Tables S1 and S2). Blood cultures were missing for five (7\%) subjects, and urine cultures were missing for 21 (28\%) subjects (Supplemental Table S1). Within the study cohort, 12 (16\%) patients were recruited from the ICU and 64 (84\%) from the HDUs. The cohort comprised $39 \%$ male and $46 \%$ postoperative patients, and the median age was 30 (IQR: 23-40) years. The most common admission diagnoses were postoperative monitoring, shock (e.g., hemorrhagic or distributive), neurological injury, and diabetic ketoacidosis (Supplemental Table S3). HIV prevalence was $13 \%$.

Eighteen patients $(24 \%)$ had sepsis based on a qSOFA score of two or more, 50 patients $(66 \%)$ had sepsis based on 


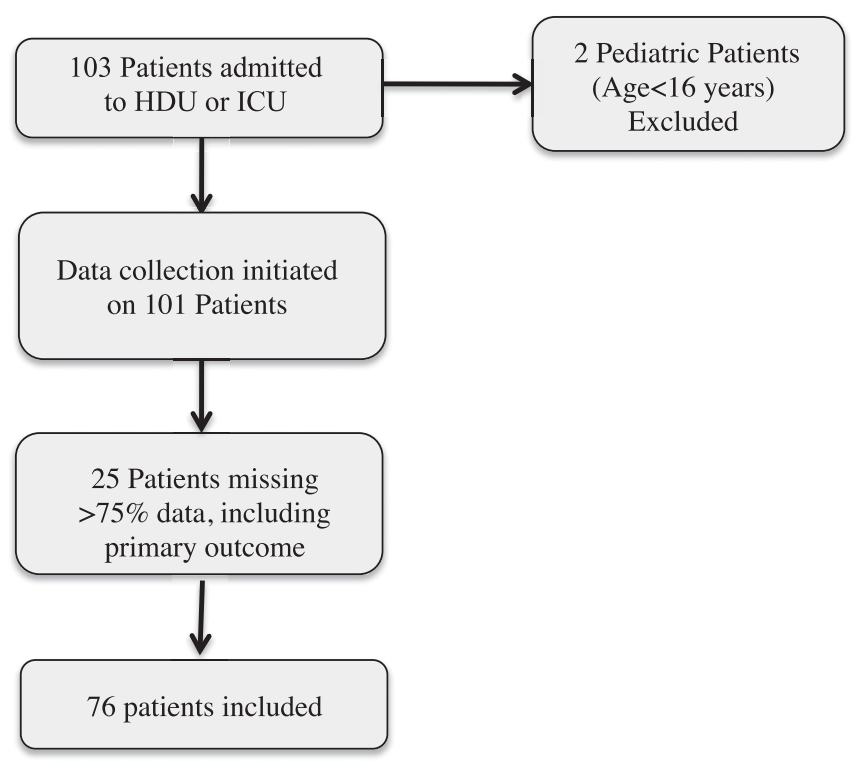

FIGURE 1. Flowchart of patient selection for analysis.

clinical suspicion of systemic infection, and 12 patients (16\%) had sepsis based on the qSOFA score plus clinical suspicion of systemic infection at ICU or HDU admission. Based on these three definitions of sepsis, hospital mortality ranged from $50 \%$ to $75 \%$ for those with sepsis versus $12 \%$ to $26 \%$ for those without sepsis. The difference in hospital mortality was significant when using clinical suspicion of systemic infection or qSOFA score $\geq 2$ plus clinical suspicion of systemic

TABLE 1

Clinical characteristics of critically ill cohort at a referral hospital in Malawi, stratified by three definitions of sepsis applicable in a low-resource environment

\begin{tabular}{|c|c|c|c|c|c|c|c|}
\hline Sepsis definition: & $\begin{array}{c}\text { qSOFA } \geq 2 \\
(n=18)\end{array}$ & $\begin{array}{c}\text { No sepsis } \\
(n=58)\end{array}$ & $\begin{array}{c}\text { Clinically suspected } \\
\text { systemic infection }(n=50)\end{array}$ & $\begin{array}{c}\text { No sepsis } \\
(n=26)\end{array}$ & $\begin{array}{c}\text { qSOFA } \geq 2 \text { plus } \\
\text { clinically suspected } \\
\text { systemic infection }(n=12)\end{array}$ & $\begin{array}{c}\text { No sepsis } \\
(n=64)\end{array}$ & $\begin{array}{c}\text { Total } \\
(n=76)\end{array}$ \\
\hline Male, $n(\%)$ & $7(39)$ & $23(40)$ & $16(32)$ & $14(54)$ & $3(25)$ & $27(42)$ & $30(39)$ \\
\hline Age (years), median (IQR) & $33(21-40)$ & $30(23-42)$ & $28(22-37)^{\star}$ & $36(30-49)^{\star}$ & $31(19-53)$ & $30(23-40)$ & $30(23-40)$ \\
\hline \multicolumn{8}{|l|}{ Study unit } \\
\hline ICU & $2(11)$ & $9(16)$ & $11(22)^{*}$ & $0(0)^{*}$ & $2(17)$ & $9(14)$ & $12(16)$ \\
\hline HDU-Medicine & $5(28)$ & $17(30)$ & $18(37)^{\star}$ & $4(15)^{\star}$ & $4(33)$ & $18(29)$ & $22(29)$ \\
\hline HDU-OBGyn & $6(33)$ & $18(32)$ & $15(31)^{*}$ & $9(34)^{\star}$ & $4(33)$ & $20(32)$ & $24(32)$ \\
\hline HDU-Surgery & $5(28)$ & $13(23)$ & $5(10)^{*}$ & $13(50)^{*}$ & $2(17)$ & $16(25)$ & $18(24)$ \\
\hline $\begin{array}{l}\text { Surgery preceding } \\
\text { HDU/ICU admission, } \\
n(\%)\end{array}$ & $8(44)$ & $26(46)$ & $23(46)$ & $11(46)$ & $5(42)$ & $29(47)$ & $34(46)$ \\
\hline \multicolumn{8}{|l|}{$\begin{array}{l}\text { Vital signs at HDU/ICU } \\
\text { admission }\end{array}$} \\
\hline $\begin{array}{l}\text { Heart rate, median } \\
\text { (IQR) }\end{array}$ & $119(80-139)$ & $107(88-133)$ & $116(93-143)$ & $97(79-117)$ & $120(100-144)$ & $107(88-133)$ & $109(88-134)$ \\
\hline $\begin{array}{l}\text { Respiratory rate, } \\
\text { median (IQR) }\end{array}$ & $28(22-31)$ & $22(18-28)$ & $24(18-30)$ & $24(19-30)$ & $27(22-32)$ & $23(18-30)$ & $24(18-30)$ \\
\hline $\begin{array}{l}\text { Mean arterial } \\
\text { pressure }(\mathrm{mmHg}) \\
\text { median }(\mathrm{IQR})\end{array}$ & $72(64-87)^{\star}$ & $90(79-106)^{\star}$ & $86(69-99)^{*}$ & $92(77-113)^{\star}$ & $67(57-89)^{\star}$ & $88(78-104)^{\star}$ & $86(76-100)$ \\
\hline $\begin{array}{l}\text { Presence of fever } \\
\left(>38.4^{\circ} \mathrm{C}\right) \text { during } \\
\text { course, } n(\%)\end{array}$ & $8(44)$ & $20(36)$ & $23(46)$ & $5(22)$ & $7(58)$ & $21(34)$ & $28(38)$ \\
\hline \multicolumn{8}{|l|}{$\begin{array}{l}\text { Laboratory } \\
\text { measurements }\end{array}$} \\
\hline HIV positive, $n(\%)$ & $2(15)$ & $6(13)$ & $7(17)$ & $1(6)$ & $2(22)$ & $6(12)$ & $8(13)$ \\
\hline Malaria, $n(\%)$ & $4(24)$ & $12(22)$ & $11(23)$ & $5(22)$ & $2(18)$ & $14(23)$ & $16(23)$ \\
\hline $\begin{array}{l}\text { Leukocyte count, } \\
\text { median (IQR) }\end{array}$ & $12(6-19)$ & $9(6-13)$ & $10(5-15)$ & $8(7-14)$ & $12(6-19)$ & $9(6-14)$ & $9(6-15)$ \\
\hline \multicolumn{8}{|l|}{ Outcomes } \\
\hline Hospital death, $n(\%)$ & $9(50)$ & $15(26)$ & $21(42)^{\star}$ & $3(12)^{*}$ & $9(75)^{\star}$ & $15(23)^{\star}$ & $24(32)$ \\
\hline
\end{tabular}


infection as the definition of sepsis (Table 1, Supplemental Appendix Table 1). In multivariate analysis of sepsis status using the three definitions (qSOFA $\geq 2$, clinical suspicion of infection at ICU/HDU admission, and qSOFA score $\geq 2$ plus clinical suspicion of infection respectively), sepsis was associated with an odds ratio for hospital mortality of 2.2 (95\% Cl: 0.4-11.3; $P=0.363), 12.3$ (95\% Cl: $1.2-123.5 ; P=$ $0.032)$, and $5.7(95 \% \mathrm{Cl}: 0.8-38.8 ; P=0.075)$, respectively (Table 2).

Most patients (74\%) had been started on antibiotics at or before ICU or HDU admission, before cultures were drawn. The majority of those on antibiotics were treated with ceftriaxone (98\%) and metronidazole (68\%). For those on ceftriaxone, the median number of days of treatment before cultures were drawn was 2 days (IQR: 1-3, range 0-15 days), and the median total treatment length was 7 days (IQR: 4-9). For those on metronidazole, the median number of days of treatment before cultures were drawn was 1 day (IQR: 0-2, range $0-8$ days), and the median total treatment length was 6 days (IQR: $2-9$ ).

Four blood cultures (5\%) were positive, two from patients who met all three definitions of sepsis and two from patients who were suspected of systemic infection but did not meet the qSOFA criteria (Table 3). All blood bacterial isolates were multidrug resistant. Hospital mortality among patients with positive blood cultures was $100 \%$.

Nine patients had positive urine cultures, five of which had urinary tract infections. Three of the urinary tract infections were associated with sepsis based on the qSOFA and suspected infection status. Two of these urinary tract infections were associated with multidrug-resistant bacteria; one of these patients died in hospital and one was lost to follow-up. Two additional urinary tract infections were associated with sepsis based on the suspected infection status alone, but these were not associated with multidrug-resistant pathogens (Table 4). Mycobacterial cultures were negative for all patients.

\section{DISCUSSION}

In this study of a critically ill cohort in Malawi, we demonstrate a prevalence of sepsis ranging from $16 \%$ to $24 \%$ to $66 \%$ based on three definitions of sepsis applicable in this environment. In-hospital mortality was higher for patients with sepsis and ranged from $42 \%$ to $75 \%$. Sepsis is a leading cause of morbidity and mortality worldwide, particularly in sub-Saharan Africa where resources are limited. ${ }^{15,16}$ These data demonstrate higher sepsis mortality than that seen in high-income settings. This may be not only due to the inadequate healthcare infrastructure in Malawi but also due to antimicrobial resistance or a high local prevalence of infectious diseases that affect immune function. There is an urgent need to direct global resources toward improving sepsis care in this region, particularly given its growing population and urbanization. ${ }^{17,18}$

Sepsis is a heterogeneous clinical syndrome, and its precise definition is a challenge. The Third International Consensus Definitions for Sepsis and Septic Shock (Sepsis-3) noted in 2016 that there are no simple, unambiguous biomarkers or criteria that uniquely identify sepsis patients. ${ }^{19}$ However, an operational definition is imperative to define the prevalence and outcomes of sepsis so that health care resources can be directed appropriately. This conundrum is especially pronounced in LMICs, where even the most basic diagnostic adjuncts are often absent. Based on the Sepsis-3 recommendations, we determined that the qSOFA score was one of the few metrics feasible for our study site. Although the score has been discussed as a ward screening tool rather than a diagnostic tool, in our study setting where care is so different from high-income settings, it was a reasonable choice. ${ }^{20}$ Notably, the full SOFA score would not be feasible in our setting, and the systemic inflammatory response syndrome criteria have not been shown to discriminate for poor outcomes. ${ }^{19}$ We also evaluated the clinical suspicion of systemic infection, a subjective staff assessment of sepsis status, which is a very common regional measure of sepsis and provides insight into staffing perceptions of sepsis symptoms. These definitions of sepsis yielded widely different estimates of the prevalence of sepsis. This demonstrates how important a feasible, unified definition for sepsis is to understanding the prevalence of sepsis in LMICs and across the sub-Saharan region.

The high sepsis-related mortality in this study is consistent with global reports from the sub-Saharan region. High mortality has been attributed to widespread malnutrition, poverty, and a high incidence of bacterial, parasitic, and HIV

TABLE 2

Multivariable analyses of odds ratio for hospital mortality using three definitions of sepsis

\begin{tabular}{|c|c|c|c|c|c|c|}
\hline Sepsis definition: & qSOFA $\geq 2$ & $P$-value & $\begin{array}{l}\text { Clinically suspected } \\
\text { systemic infection }\end{array}$ & $P$-value & $\begin{array}{l}\text { qSOFA } \geq 2 \text { plus clinically } \\
\text { suspected systemic infection }\end{array}$ & $P$-value \\
\hline Sepsis & $2.2(0.4-11.3)$ & 0.363 & $12.3(1.2-123.5)$ & 0.032 & $5.7(0.8-38.8)$ & 0.075 \\
\hline Gender & - & - & - & - & - & - \\
\hline Female & - & & Reference & & - & \\
\hline Male & - & & $3.5(0.7-17.3)$ & 0.119 & - & \\
\hline Age & - & - & $1.1(0.9-1.1)$ & 0.051 & - & - \\
\hline \multicolumn{7}{|l|}{ Vital signs at HDU/ICU admission } \\
\hline Heart rate & $1.0(0.9-1.0)$ & 0.075 & $1.0(0.9-1.1)$ & 0.060 & $1.0(0.9-1.0)$ & 0.085 \\
\hline Mean arterial pressure & $0.9(0.9-1.0)$ & 0.175 & $0.9(0.9-1.0)$ & 0.092 & $0.9(0.9-1.0)$ & 0.185 \\
\hline $\begin{array}{l}\text { Presence of fever }\left(>38.4^{\circ} \mathrm{C}\right) \\
\text { during course }\end{array}$ & $2.6(0.7-9.8)$ & 0.166 & $5.1(1.2-21.3)$ & 0.024 & $2.3(0.6-9.3)$ & 0.237 \\
\hline \multicolumn{7}{|l|}{ Laboratory measurements } \\
\hline HIV positive & $7.8(1.2-48.5)$ & 0.028 & - & - & $7.3(1.1-48.3)$ & 0.039 \\
\hline
\end{tabular}

$\mathrm{C}=$ Celsius; HDU = high-dependency unit; ICU = intensive care unit; IQR = interquartile range; $\mathrm{mmHg}=$ millimeters of mercury; OBGyn = obstetrics and gynecology; qSOFA = quick sequential organ failure assessment. Original variables included in the multivariable model before backward selection included the following: sepsis status, gender, age, postoperative status, heart rate at ICU/ $\mathrm{HDU}$ admission, mean arterial pressure at ICU/HDU admission, presence of fever during ICU/HDU course, HIV status, and malaria status. Continuous variables were analyzed according to increments of 1 unit (e.g., 1 year for age, one beat per minute for heart rate, $1 \mathrm{mmHg}$ for mean arterial pressure, and $1^{\circ} \mathrm{C}$ for temperature). All models were statistically significant at $P<0.05$. 
TABLE 3

Positive blood culture results with sepsis status based on three definitions and fever status* $, \uparrow, \ddagger$

\begin{tabular}{|c|c|c|c|c|c|c|c|c|}
\hline Patient & $\begin{array}{c}\text { Sepsis } \\
\text { basedon } \\
\text { qSOFA } \\
\geq 2\end{array}$ & $\begin{array}{l}\text { Sepsis based on } \\
\text { clinically } \\
\text { suspected } \\
\text { systemic } \\
\text { infection }\end{array}$ & $\begin{array}{l}\text { Sepsis based on } \\
\text { qSOFA } \geq 2 \text { and } \\
\text { clinically } \\
\text { suspected } \\
\text { systemic } \\
\text { infection }\end{array}$ & Fever & $\begin{array}{l}\text { Organisms } \\
\text { isolated }\end{array}$ & Sensitive to & $\begin{array}{l}\text { Intermediate } \\
\text { sensitivity }\end{array}$ & Resistant to \\
\hline A & Yes & Yes & Yes & Yes & $\begin{array}{l}\text { Proteus } \\
\quad \text { mirabilis }\end{array}$ & Imipenem & - & $\begin{array}{l}\text { Amoxicillin-clavulanic acid, } \\
\text { ampicillin, cefotaxime, } \\
\text { ceftriaxone, chloramphenicol, } \\
\text { ciprofloxacin, gentamicin, } \\
\text { nalidixic acid, and } \\
\text { trimethoprim-sulfamethoxazole }\end{array}$ \\
\hline \multirow[t]{2}{*}{ B } & Yes & Yes & Yes & Yes & $\begin{array}{r}\text { Klebsiella } \\
\text { oxytoca }\end{array}$ & $\begin{array}{l}\text { Chloramphenicol, } \\
\text { ciprofloxacin, } \\
\text { and nalidixic } \\
\text { acid }\end{array}$ & - & $\begin{array}{l}\text { Amoxicillin-clavulanic acid, } \\
\text { ampicillin, cefotaxime, } \\
\text { ceftriaxone, gentamicin, and } \\
\text { trimethoprim-sulfamethoxazole }\end{array}$ \\
\hline & & & & & Escherichia coli & Gentamicin & $\begin{array}{l}\text { Chloramphenicol, } \\
\text { ciprofloxacin, } \\
\text { and nalidixic } \\
\text { acid }\end{array}$ & $\begin{array}{l}\text { Amoxicillin-clavulanic acid, } \\
\text { ampicillin, cefotaxime, } \\
\text { ceftriaxone, and } \\
\text { trimethoprim-sulfamethoxazole }\end{array}$ \\
\hline C & No & Yes & No & No & $\begin{array}{l}\text { Coagulase- } \\
\text { negative } \\
\text { Staphylococcus }\end{array}$ & $\begin{array}{l}\text { Ciprofloxacin and } \\
\text { cefoxitin }\end{array}$ & - & $\begin{array}{l}\text { Cefoxitin, gentamicin, and } \\
\text { oxacillin }\end{array}$ \\
\hline D & No & Yes & No & Yes & Candida glabrata & $\mathrm{NR}^{*}$ & $\mathrm{NR}^{*}$ & $\mathrm{NR}^{\star}$ \\
\hline
\end{tabular}

${ }^{*}$ Sensitivities for Candida were not reported by laboratory.

† Mycobacterial cultures were negative for all patients.

$\ddagger$ Blood cultures were missing for five $(7 \%)$ subjects, and urine cultures were missing for 21 (28\%) subjects.

infections. ${ }^{21}$ It is also likely due to a lack of resources. In Malawi, there is only an estimated 1.7 ICU beds per million population. ${ }^{22}$ In this study, most patients diagnosed with sepsis were located in the HDU rather than the ICU. This may reflect the shortage of ICU beds, a missed diagnosis of sepsis, or a combination of these factors. The lack of resources extends beyond ICU availability. In a recent published survey, only a minority of sub-Saharan African respondents could implement sepsis resuscitation recommendations related to lactate measurement and antibiotic administration. ${ }^{23}$ Although most drugs of the WHO Essential Medicine List ${ }^{24}$ can be theoretically purchased in Malawi, only a few of these drugs are immediately available for inpatients at our study site, one of the country's largest public referral hospitals. Aside from shortages in vital medications (e.g., epinephrine, norepinephrine, dopamine, broad-spectrum antibiotics, and furosemide), sub-Saharan African healthcare systems also have to contend with low-quality and/or "fake" drugs. ${ }^{25}$ Finally, there is a shortage of personnel with advanced training in sepsis management. In Malawi, where clinical officers and nurses provide the majority of health care, $45 \%$ of workforce positions are vacant. ${ }^{26}$ With so few human resources, the initial signs of sepsis may easily go unrecognized.

Antibacterial resistance is known to increase the morbidity, mortality, and cost of treatment for sepsis. ${ }^{27-30}$ All blood culture bacterial isolates in this study were multidrug resistant, and the urinary tract infections associated with sepsis by the qSOFA criteria were associated with resistant bacteria. The prevalence of antimicrobial-resistant pathogens is notable and demonstrates that antibiotic resistance is a global phenomenon. This trend has been confirmed in several other single-center studies on the prevalence of multidrug-resistant pathogens in African countries. ${ }^{31-33}$ Future research would benefit from collaboration between single centers to form regional microbiology registries in a united effort against antimicrobial resistance.
Antibiotic stewardship is a challenging proposition in LMICs, but it is critical to public health. In sub-Saharan Africa, people are using antibiotics more frequently, in part due to urbanization, lower costs of antibiotics, and poor control of over-the-counter drug sales. ${ }^{34-36}$ In hospitals, antimicrobial stewardship is challenging secondary to a lack of trained pharmacy personnel, lack of laboratory infrastructure to guide the choice of antimicrobials, and the ethical dilemma of delaying or denying potentially effective antimicrobial treatment. A recent study from Tanzania demonstrated that microbiologic cultures were only obtained from $17 \%$ of patients treated with antibiotics; only $15 \%$ of antibiotic treatment regimens met the national guidelines. ${ }^{37}$ These challenges were also reflected in this study: despite prospective data collection, it was exceedingly difficult to obtain cultures before staff administered antibiotics; changing antibiotics based on culture results was an unfamiliar concept; and antibiotic treatment is generally continued until patient discharge, regardless of culture results. The results from Tanzania and our experience reflect the need for ongoing education and infrastructure to support antimicrobial stewardship. Otherwise, these factors will continue to drive the emergence and spread of multidrug-resistant organisms in community and hospital settings. $^{38}$

To attenuate sepsis-related morbidity and mortality in subSaharan Africa, attempts have been made to introduce sepsis management protocols to the critical care setting. These interventions are based on the original "early, goal-directed" care described by Rivers et al. ${ }^{39}$ but modified for sub-Saharan Africa because of logistical constraints, such as the lack of invasive monitoring and critical care facilities. However, blind adoption of established interventions from high-income settings may prove to be ineffective or even harmful in LMICs, as demonstrated by two recent trials. ${ }^{40,41}$ Trials of protocolized care have not yet addressed the key issue of antimicrobial stewardship and resistance. 
SEPSIS AMONG CRITICALLY ILL PATIENTS IN MALAWI

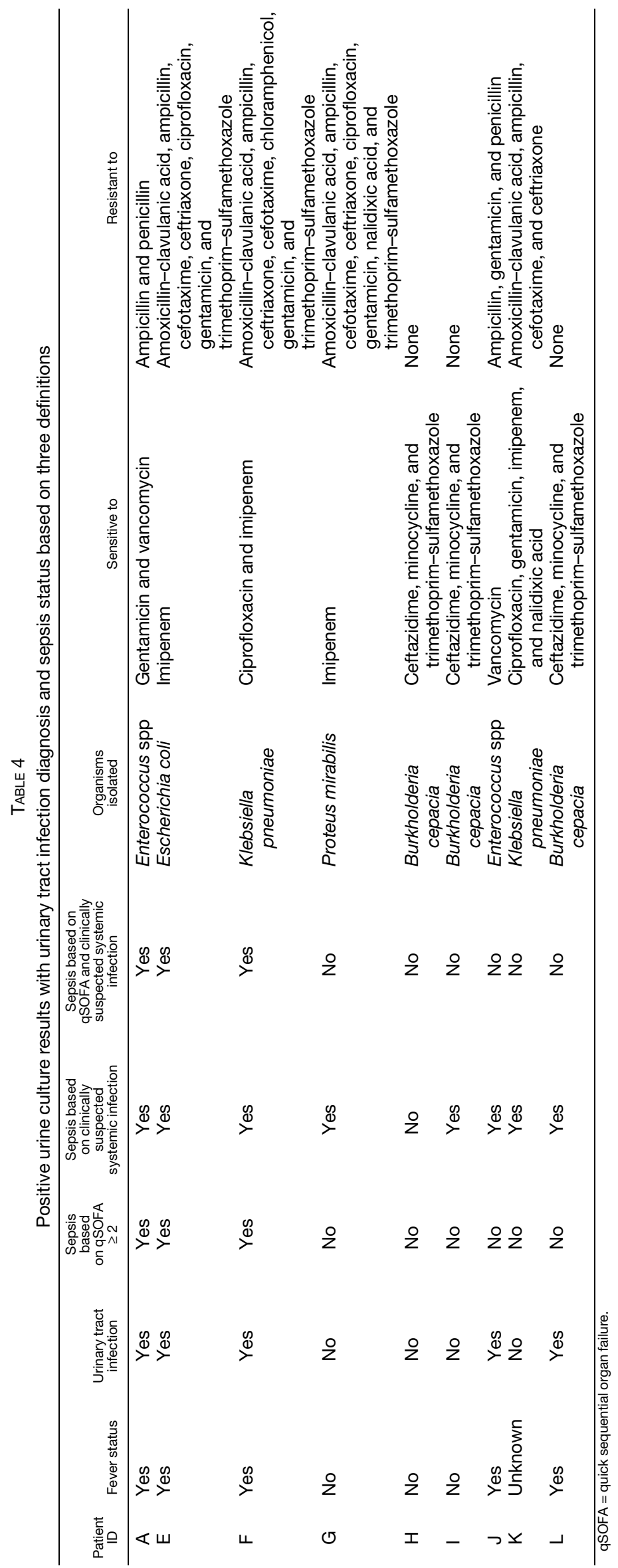


This study highlights the challenges of sepsis diagnosis and management in a LMIC. The strengths of this study are its specific operational definitions of sepsis, the granularity of the clinical data collected within a region in which data collection is known to be prohibitively difficult, and the inclusion of patients outside of the ICU. This study contributes to the literature gap in characterizing sepsis in sub-Saharan African hospitals and also toward understanding the challenges LMICs will face in treating sepsis. This study has several limitations. First, this is a single-center study conducted over a relatively short time period, which limited the sample size. Second, we excluded 25 patients for missing data. This limitation demonstrates the lack of infrastructure and the difficulties of data collection at this site. Third, it is likely that some patients on the general wards had critical illness that we did not capture. Fourth, we assessed for sepsis at admission to ICU or HDU, rather than a longitudinal assessment of vital signs.

Finally, we had several laboratory limitations. We were unable to take more than one peripheral blood culture per patient and could not take anaerobic, cerebrospinal, or pulmonary cultures. Given the difficulty of cultivating Mycobacterium in vitro, ${ }^{42}$ we acknowledge that this study may have missed mycobacterial sepsis. In addition, because antibiotic use was often initiated before collection of cultures, some cultures could have been false negatives. For all of these reasons, our results likely underestimate the prevalence of sepsis in this population.

\section{CONCLUSION}

In Malawi, sepsis-related mortality is exceedingly high. We show a $42-75 \%$ mortality following sepsis based on different definitions, and bacterial isolates from septic patients raise concern for a high incidence of multidrug-resistant organisms. Investments in laboratory infrastructure and antibiotic stewardship are critical to addressing sepsis-related mortality in LMICs.

Received August 15, 2019. Accepted for publication February 27, 2020.

Published online April 27, 2020.

Note: Supplemental tables and appendix appear at www.ajtmh.org.

Acknowledgments: We thank L. P. for presenting an abstract of this work at the 2019 annual meeting of the American College of Surgeons and the staff of Kamuzu Central Hospital for their cooperation and contributions to data collection.

Financial support: This study was supported in part by the NIH Fogarty International Center Postdoctoral Research Fellowship to M. P. and by the Friedman Award from Columbia University College of Physicians and Surgeons to L. O.

Disclaimer: The contents of the manuscript are solely the responsibility of the authors and do not necessarily reflect the official views of the funding agencies.

Disclosure: The study protocol was approved by the National Health Sciences Research Council of Malawi and the Institutional Review Boards of the American universities with which the study was affiliated, and the requirement for written informed consent was waived by all ethics oversight bodies. The data used and analyzed during the current study are available from the corresponding author on reasonable request.

Authors' addresses: Meghan Prin, Department of Anesthesiology, University of Colorado School of Medicine, Aurora, CO, E-mail: meghan.prin@cuanschutz.edu. Lauren Onofrey, Department of
Internal Medicine, University of Washington School of Medicine, Seattle, WA, E-mail: lonofrey@uw.edu. Laura Purcell and Anthony Charles, Department of Surgery, University of North Carolina at Chapel Hill, Chapel Hill, NC, E-mails: laura.purcell@unchealth.unc.edu and anthony_charles@med.unc.edu. Clement Kadyaudzu, Department of Anesthesiology, Kamuzu Central Hospital, Lilongwe, Malawi, E-mail: kadyauzdu@yahoo.com.

\section{REFERENCES}

1. Fleischmann C, Scherag A, Adhikari NK, Hartog CS, Tsaganos T, Schlattmann P, Angus DC, Reinhart K, International Forum of Acute Care Trialists, 2016. Assessment of global incidence and mortality of hospital-treated sepsis. Current estimates and limitations. Am J Respir Crit Care Med 193: 259-272.

2. Tadesse BT, Ashley EA, Ongarello S, Havumaki J, Wijegoonewardena M, Gonzalez IJ, Dittrich S, 2017. Antimicrobial resistance in Africa: a systematic review. BMC Infect Dis 17: 616.

3. Essack SY, Desta AT, Abotsi RE, Agoba EE, 2017. Antimicrobial resistance in the WHO African region: current status and roadmap for action. J Public Health (Oxf) 39: 8-13.

4. Esimone C, Nworu C, Udeogaranya O, 2007. Utilization of antimicrobial agents with and without prescription by out-patients in selected pharmacies in southeastern Nigeria. Pharm World Sci 29: 655-660.

5. Mukonzo J, Namuwenge P, Okure G, Mwesige B, Namusisi O, Mukanga D, 2013. Over-the-counter suboptimal dispensing of antibiotics in Uganda. J Multidiscip Healthc 6: 303-310.

6. UN, 2016. Human Development Reports. United Nations Development Programme. Available at: http://hdr.undp.org/en/ countries/profiles/MWI. Accessed May 18, 2018.

7. Gregson J, 2017. The richest countries in the World: international monetary fund, world economic outlook database. Global Finance.

8. Pavlinac PB, Lokken EM, Walson JL, Richardson BA, Crump JA, John-Stewart GC, 2016. Mycobacterium tuberculosis bacteremia in adults and children: a systematic review and metaanalysis. Int J Tuberc Lung Dis 20: 895-902.

9. Cummings M, ODonnell M, 2015. Inverting the pyramid: awareness of mycobacterial sepsis in sub-Saharan Africa. Int $J$ Tuberc Lung Dis 19: 1128-1134.

10. Prin M, Pan S, Kadyaudzu C, Li G, Charles A, 2018. Development of a malawi intensive care mortality risk evaluation (MIME) model, a prospective cohort study. Int J Surg 60: 60-66.

11. Clinical and Laboratory Standards Institute, 2017. Performance Standards for Antimicrobial Susceptibility Testing, 27th edition. Wayne, PA: Clinical and Laboratory Standards Institute.

12. Magiorakos AP et al., 2012. Multidrug-resistant, extensively drugresistant and pandrug-resistant bacteria: an international expert proposal for interim standard definitions for acquired resistance. Clin Microbiol Infect 18: 268-281.

13. $\mathrm{MoH}, 2009$. Malawi Standard Treatment Guidelines, 4th edition. Available at: http://apps.who.int/medicinedocs/documents/ s18801en/s18801en.pdf. Accessed July 1, 2019.

14. Huson MAM, Katete C, Chunda L, Ngoma J, Wallrauch C, Heller T, van der Poll T, Grobusch MP, 2017. Application of the qSOFA score to predict mortality in patients with suspected infection in a resource-limited setting in Malawi. Infection 45: 893-896.

15. Rudd KE, Kissoon N, Limmathurotsakul D, Bory S, Mutahunga B, Seymour CW, Angus DC, West TE, 2018. The global burden of sepsis: barriers and potential solutions. Crit Care 22: 232.

16. Becker JU, Theodosis C, Jacob ST, Wira CR, Groce NE, 2009. Surviving sepsis in low-income and middle-income countries: new directions for care and research. Lancet Infect Dis 9: 577-582.

17. Annez PC, Linn JF, 2010. An agenda for research on urbanization in developing countries: a summary of findings from a scoping exercise. World Bank

18. Austin S et al., 2014. Access to urban acute care services in highvs. middle-income countries: an analysis of seven cities. Intensive Care Med 40: 342-352.

19. Singer $M$ et al., 2016. The third international consensus definitions for sepsis and septic shock (sepsis-3). JAMA 315: 801-810. 
20. Rudd KE et al., 2018. Association of the quick sequential (sepsisrelated) organ failure assessment (qSOFA) score with excess hospital mortality in adults with suspected infection in low- and middle-income countries. JAMA 319: 2202-2211.

21. Cheng AC, West TE, Limmathurotsakul D, Peacock S, 2008. Strategies to reduce mortality from bacterial sepsis in adults in developing countries. PLoS Med 5: 1173-2117.

22. Manda-Taylor L, Mndolo S, Baker T, 2017. Critical care in Malawi: the ethics of beneficence and justice. Malawi Med $J$ 29: 268-271.

23. Baelani I, Jochberger S, Laimer T, Otieno D, Kabutu J, Wilson I, Baker T, Dünser M, 2011. Availability of critical care resources to treat patients with severe sepsis or septic shock in Africa: a self-reported, continent-wide survey of anaesthesia providers. Crit Care 15: R10.

24. Laing R, Waning B, Gray A, Ford N, 't Hoen E, 2003. 25 years of the WHO essential medicine lists: progress and challenges. Lancet 361: 1723-1729.

25. Raufu A, 2003. India agrees to help Nigeria tackle the import of fake drugs. BMJ 326: 1234.

26. Government of the Republic of Malawi, 2016. 2016 Government Of the Republic of Malawi, Health Sector Strategic Plan II (2017-2022). Available at: http://www.nationalplanningcycles. org/sites/default/files/planning_cycle_repository/malawi/ health_sector_strategic_plan_ii_030417_smt_dps.pdf, 27. Accessed August 1, 2019.

27. Zilberberg MD, Shorr AF, Micek ST, Vazquez-Guillamet C, Kollef $\mathrm{MH}, 2014$. Multi-drug resistance, inappropriate initial antibiotic therapy and mortality in gram-negative severe sepsis and septic shock: a retrospective cohort study. Crit Care 18: 596.

28. Naylor NR, Atun R, Zhu N, Kulasabanathan K, Silva S, Chatterjee A, Knight GM, Robotham JV, 2018. Estimating the burden of antimicrobial resistance: a systematic literature review. Antimicrob Resist Infect Control 7: 58.

29. Zilberberg MD, Nathanson BH, Sulham K, Fan W, Shorr AF, 2017. 30-day readmission, antibiotics costs and costs of delay to adequate treatment of Enterobacteriaceae UTI, pneumonia, and sepsis: a retrospective cohort study. Antimicrob Resist Infect Control 6: 124.

30. Zilberberg MD, Nathanson BH, Sulham K, Fan W, Shorr AF, 2017. Carbapenem resistance, inappropriate empiric treatment and outcomes among patients hospitalized with Enterobacteriaceae urinary tract infection, pneumonia and sepsis. BMC Infect Dis 17: 279.
31. Mhondoro M, Ndlovu N, Bangure D, Juru T, Gombe NT, Shambira G, Nsubuga P, Tshimanga M, 2019. Trends in antimicrobial resistance of bacterial pathogens in Harare, Zimbabwe, 2012-2017: a secondary dataset analysis. BMC Infect Dis 19: 746.

32. Wangai FK, Masika MM, Lule GN, Karari EM, Maritim MC, Jaoko WG, Museve B, Kuria A, 2019. Bridging antimicrobial resistance knowledge gaps: the east African perspective on a global problem. PLoS One 14: e0212131.

33. Mauchaza K, Madzimbamuto FD, Waner S, 2016. Methicillinresistant Staphylococcus aureus in Zimbabwe. Ghana Med J 50: 68-71.

34. Klein EY, Van Boeckel TP, Martinez EM, Pant S, Gandra S, Levin SA, Goossens H, Laxminarayan R, 2018. Global increase and geographic convergence in antibiotic consumption between 2000 and 2015. Proc Natl Acad Sci USA 115: E3463-E70.

35. Vialle-Valentin C, LeCates R, Zhang F, Desta A, Ross-Degnan D, 2012. Predictors of antibiotic use in African communities: evidence from medicines household surveys in five countries. Trop Med Int Health 17: 211-222.

36. Ayukekbong J, Ntemgwa M, Atabe A, 2017. The threat of antimicrobial resistance in developing countries: causes and control strategies. Antimicrob Resist Infect Control 6: 47.

37. Haldeman MS et al., 2020. Evaluation of antimicrobial utilization and concordance with national guidelines at a tertiary hospital in the southern highland zone of Tanzania. Am J Trop Med Hyg 102: 370-376.

38. Holmes AH, Moore L, Sundsfjord A, Steinbakk M, Regmi S, Karkey A, Guerin P, Piddock L, 2016. Understanding the mechanisms and drivers of antimicrobial resistance. Lancet 387: 176-187.

39. Rivers E, Nguyen B, Havstad S, Ressler J, Muzzin A, Knoblich B, Peterson E, Tomlanovich M, 2001. Early goal-directed therapy in the treatment of severe sepsis and septic shock. $N$ Engl $J$ Med 345: 1368-1377.

40. Maitland $\mathrm{K}$ et al., 2011. Mortality after fluid bolus in African children with severe infection. N Engl J Med 364: 2483-2495.

41. Andrews B, Semler MW, Muchemwa L, Kelly P, Lakhi S, Heimburger DC, Mabula C, Bwalya M, Bernard GR, 2017. Effect of an early resuscitation protocol on in-hospital mortality among adults with sepsis and hypotension: a randomized clinical trial. JAMA 318: 1233-1240.

42. Dunn JJS JR, Revell PA, 2016. Laboratory diagnosis of Mycobacterium tuberculosis infection and disease in children. $J$ Clin Microbiol 54: 1434-1441. 